\title{
Structure of the $\mathrm{Ca}^{2+}$-dependent PP2A heterotrimer and insights into $\mathrm{Cdc} 6$ dephosphorylation
}

\author{
Nathan Wlodarchak ${ }^{1, *}$, Feng Guo ${ }^{1, *}$, Kenneth A Satyshur ${ }^{1}$, Li Jiang ${ }^{1}$, Philip D Jeffrey ${ }^{2}$, Tingwan Sun ${ }^{3}$, \\ Vitali Stanevich ${ }^{1}$, Marc C Mumby ${ }^{3}$, Yongna Xing ${ }^{1}$ \\ ${ }^{I}$ McArdle Laboratory, Department of Oncology, University of Wisconsin-Madison, School of Medicine and Public Health, Madi- \\ son, WI, USA; ${ }^{2}$ Department of Molecular Biology, Princeton University, Princeton, NJ, USA; ${ }^{3}$ Department of Pharmacology, Uni- \\ versity of Texas Southwestern Medical Center, Dallas, TX, USA
}

The B"/PR72 family of protein phosphatase 2A (PP2A) is an important PP2A family involved in diverse cellular processes, and uniquely regulated by calcium binding to the regulatory subunit. The PR70 subunit in this family interacts with cell division control 6 (Cdc6), a cell cycle regulator important for control of DNA replication. Here, we report crystal structures of the isolated PR72 and the trimeric PR70 holoenzyme at a resolution of 2.1 and $2.4 \AA$, respectively, and in vitro characterization of Cde6 dephosphorylation. The holoenzyme structure reveals that one of the PR70 calcium-binding motifs directly contacts the scaffold subunit, resulting in the most compact scaffold subunit conformation among all PP2A holoenzymes. PR70 also binds distinctively to the catalytic subunit near the active site, which is required for PR70 to enhance phosphatase activity toward Cdc6. Our studies provide a structural basis for unique regulation of $B^{\prime \prime} / P R 72$ holoenzymes by calcium ions, and suggest the mechanisms for precise control of substrate specificity among PP2A holoenzymes.

Keywords: protein phosphatase 2A; regulatory subunit; calcium-binding motif; Cdc6; cell cycle Cell Research (2013) 23:931-946. doi:10.1038/cr.2013.77; published online 11 June 2013

\section{Introduction}

The tight control of reversible protein phosphorylation and dephosphorylation is crucial for the regulation of cellular function $[1,2]$. Protein phosphatase 2A (PP2A) is a major Ser/Thr phosphatase in all eukaryotic cells that participates in many cellular processes via the formation of diverse trimeric holoenzymes. Each holoenzyme comprises of a common core enzyme formed by the scaffold (A) and catalytic (C) subunits (two isoforms each) that recruits a variable regulatory subunit derived from four different families (B/B55/PR55, B'/B56/PR61, B"/PR72, and $\mathrm{B}^{\prime \prime \prime} /$ Striatin) $[3,4]$. The regulatory subunits control substrate specificity and intracellular distribution of PP2A holoenzymes. Collectively, PP2A holoenzymes tar-

\footnotetext{
*These two authors contributed equally to this work.

Correspondence: Yongna Xing

E-mail: xing@oncology.wisc.edu

Received 23 March 2013; revised 17 April 2013; accepted 25 April 2013; published online 11 June 2013
}

get a broad array of cellular phosphoproteins $[3,4]$. The three-dimensional structures of two PP2A holoenzymes have been determined [5], but no structures are reported for the B"/PR72 and B"'/Striatin families of PP2A regulatory subunits and holoenzymes. Furthermore, tight regulation of dephosphorylation of cellular proteins remains poorly understood, as it is unclear how PP2A regulatory subunits restrict the access to the PP2A active site, allowing only selected substrates to be dephosphorylated.

Members of B"/PR72 family of PP2A regulatory subunits are involved in multiple cellular processes, including modulation of neuronal signaling [6], Wnt signaling $[7,8]$, regulation of calcium-channel phosphorylation $[9,10]$ and tumor suppression [11]. The PR72 and PR70 members of this family have been proposed to play a role in cell cycle progression by regulating the retinoblastoma and cell division control 6 (Cdc6) proteins, which are both crucial for the G1/S transition [12-14]. The precise control of Cdc6 levels is important for the correct timing of DNA replication, and helps to ensure that only one copy of the genome is synthesized during each cell cycle $[15,16]$. The N-terminal domain of Cdc6 harbors 
$\mathrm{R} X X \mathrm{~L}$ (D box) and KEN (KEN box) destruction motifs, which target Cdc6 for ubiquitination and degradation [17]. Ubiquitination is negatively regulated by the phosphorylation of serine residues at 54, 74, and 106 of Cdc6 by cyclin-dependent kinases during G1 [18]. Several lines of evidence suggest that the PR70 regulatory subunit binds to Cdc6 and plays a role in controlling its cellular level [14]. How the phosphatase activity toward Cdc6 is regulated, however, remains largely unclear.

The A subunit of PP2A contains 15 HEAT (huntingtinelongation-A subunit-TOR) repeats $[19,20]$. The catalytic subunit specifically recognizes HEAT repeats 1115 [21] and regulatory subunits from the B/PR55 and B'/
PR61 families interact with the N-terminal HEAT repeats [22-24]. The B"/PR72 family has no sequence similarity to $\mathrm{B} / \mathrm{PR} 55$ or $\mathrm{B}^{\prime} / \mathrm{PR} 61$ regulatory subunits. All members of the B"/PR72 family have two conserved EF hand calcium-binding motifs. Calcium binding was shown to enhance the interaction of these subunits with the PP2A core enzyme, and affect the phosphatase activity [6, 12, 14]. However, the lack of structural information for this family of regulatory subunits and holoenzymes has prevented mechanistic understanding of $\mathrm{PP} 2 \mathrm{~A}$ regulation by B"/PR72 regulatory subunits.

Here we report the crystal structures of the isolated PR72 and the trimeric PP2A holoenzyme containing

Table 1 Crystallographic data collection, phasing, and refinement for PR72

\begin{tabular}{|c|c|c|c|c|c|}
\hline \multicolumn{6}{|c|}{ Data collection and phasing } \\
\hline \multirow[t]{2}{*}{ Crystal } & \multicolumn{3}{|c|}{$\mathrm{B}^{\prime \prime}$ mono calcium } & \multicolumn{2}{|c|}{$\mathrm{B}^{\prime \prime}$ mono B" di } \\
\hline & \multicolumn{3}{|c|}{ Selenomethionine } & \multirow[t]{2}{*}{ Calcium native } & \multirow[t]{2}{*}{ Calcium native } \\
\hline Data set & Peak & Inflection & Remote & & \\
\hline Wavelength $(\AA)$ & 0.9790 & 0.9793 & 0.9611 & 1.100 & 1.10 \\
\hline \multirow[t]{2}{*}{ Resolution $(\AA)$} & $100-2.8$ & $100-2.8$ & $100-2.8$ & $100-2.09$ & $50-2.9$ \\
\hline & $(2.9-2.8)$ & $(2.9-2.8)$ & $(2.9-2.8)$ & $(2.18-2.09)$ & $(3.0-2.9)$ \\
\hline Unique reflections & 17,443 & 17,596 & 17,462 & 21,335 & 20,230 \\
\hline Completeness (\%) & $99.4(99.5)$ & $99.2(95.2)$ & $99.5(99.5)$ & $94.9(61.2)$ & $99.8(98.9)$ \\
\hline Redundancy & $3.7(3.6)$ & $3.6(3.4)$ & $3.7(3.6)$ & $4.7(2.3)$ & $3.6(3.1)$ \\
\hline R-symm & $0.245(0.096)$ & $0.117(0.457)$ & $0.108(0.322)$ & $0.083(0.442)$ & $0.076(0.609)$ \\
\hline$<\mathrm{I} / \mathrm{s} \mathrm{s}>$ & $11.3(3.7)$ & $7.9(1.7)$ & $9.6(2.5)$ & $10.9(1.3)$ & $11.9(1.8)$ \\
\hline \multicolumn{6}{|l|}{ Phasing } \\
\hline Figure of merit & 0.82 & & & & \\
\hline \multicolumn{6}{|l|}{$\underline{\text { Refinement }}$} \\
\hline \multirow[t]{2}{*}{ Resolution $(\AA)$} & & & & $35-2.09$ & $35-2.9$ \\
\hline & & & & $(2.20-2.09)$ & $(3.05-2.90)$ \\
\hline No. of reflections (free) & & & & $20326(1029)$ & $20215(1024)$ \\
\hline Completeness (\%) & & & & 90.5 & 99.6 \\
\hline R-factor (\%) & & & & $19.41(26.8)$ & $20.95(26.0)$ \\
\hline R-free (\%) & & & & $25.22(35.1)$ & $25.75(32.1)$ \\
\hline \multicolumn{6}{|l|}{ No. of atoms } \\
\hline Protein & & & & 2222 & 4444 \\
\hline Water & & & & 83 & 0 \\
\hline Calcium & & & & 1 & 4 \\
\hline RMSD bond lengths $(\AA)$ & & & & 0.009 & 0.011 \\
\hline RMSD bond angles $\left({ }^{\circ}\right)$ & & & & 1.122 & 0.890 \\
\hline Average B-factors $\left(\AA^{2}\right)$ & & & & 50.1 & 77.1 \\
\hline \multicolumn{6}{|l|}{ Ramachandran plot } \\
\hline Favored (\%) & & & & 97.7 & 96.0 \\
\hline Allowed (\%) & & & & 2.3 & 3.8 \\
\hline Outliers (\%) & & & & 0.0 & 0.2 \\
\hline
\end{tabular}

X-ray diffraction data were collected on one crystal. Values in parentheses are for highest-resolution shell. 
PR70, as well as in vitro characterization of the PR70 holoenzyme-mediated dephosphorylation of Cdc6. There is a distinct positioning of two PR70 EF-hand calciumbinding motifs. The first EF-hand (EF1) is located on the top surface of the holoenzyme facing the phosphatase active site and the second EF-hand (EF2) directly contacts the scaffold subunit. This architecture provides a possible explanation for the role of calcium binding in holoenzyme assembly and substrate recognition. Interestingly, PR70 contacts the catalytic subunit near the phosphatase active site distinctly different from the B' family of regulatory subunits. While PR70 enhances the phosphatase activity toward pCdc6, which relies on its interaction with the catalytic subunit, the B'/PR61 regulatory subunits markedly hindered Cdc6 dephosphorylation, likely by steric hindrance of Cdc6 entry route to the active site on the catalytic subunit. These studies fill an important gap of knowledge on the structural basis of the B"/PR70 holoenzymes, and provide important insights into tight control of Cde6 dephosphorylation.

\section{Results}

Crystallization of the PR72 subunit and trimeric PR70 holoenzyme

The recombinant full-length PR72 and PR70 subunits eluded crystallization after extensive efforts. As an alternative approach, we defined an elastase-resistant core of the PR72 subunit (residues 165-443) and this protein was readily crystallized. The structure was determined by Selenium MAD (multiwavelength anomalous dispersion) phasing and refined to $2.2 \AA$ (Table 1 ).

PP2A holoenzymes assembled with various PR72/ PR70 fragments failed to produce diffracting crystals. PR70 was previously shown to interact with the N-terminal fragment of Cdc6 $[13,14]$. The PR70 holoenzyme was thus assembled using PR70 fused to Cdc6 fragments, which led to a breakthrough in the crystallization of this holoenzyme. After extensive efforts, diffracting crystals were obtained for the PR70 holoenzyme bound to microcystin-LR (MCLR) containing PR70 (122490) fused to Cdc6 (70-90) via a flexible linker (STGNASDSSSDSSSSEGD GTV).

Structural determination by molecular replacement using the structure of PR72 and the structural models of the PP2A core enzyme from other holoenzymes (PDB codes: 2NPP (B' $\gamma 1$ holoenzyme), 3DW8 (B55 $\alpha$ holoenzyme)) was not successful, indicating that there are significant conformational differences between the PR70 holoenzyme and holoenzymes containing other regulatory subunits. The structure was eventually determined by Selenium SAD (single-wavelength anomalous disper- sion) phasing, and refined to $2.4 \AA$ with the native data set (Table 2). The electron density map identified majority of residues in all three PP2A subunits (residues 9-589 of the A subunit, residues 2-296 of PP2Ac and residues $122-478$ of the PR70 subunit).

\section{Overall structure of PR70 holoenzyme}

The structure of the PR70 holoenzyme measures 110 $\AA$ in width, $100 \AA$ in height, and $60 \AA$ in thickness (Figure 1). The interhelical loops of the A subunit serve as the docking sites for the regulatory and catalytic subunits, as seen in holoenzymes containing the B/PR55 and $\mathrm{B}^{\prime} /$

Table 2 Crystallographic data collection, phasing, and refinement for PP2A holoenzyme containing PR70

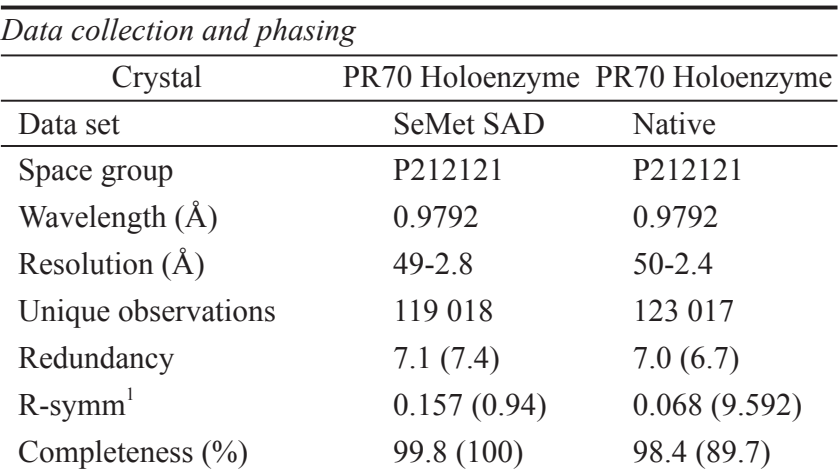

Phasing

Figure of merit $\quad 0.68$

$\underline{\text { Refinement }}$

$\begin{array}{lll}\text { Resolution }(\AA) & 49-2.8 & 50-2.4 \\ \text { No. reflections (free) } & 79333(4113) & 116785 \text { (6193) } \\ \text { Completeness (\%) } & 99.9 & 98.6 \\ \text { R-factor (\%) } & 18.3 & 17.9 \\ \text { R-free (\%) } & 24.9 & 22.8 \\ \text { Number of atoms (total) } & 19933 & 20419 \\ \text { Protein } & 19662 & 19886 \\ \text { Water } & 174 & 533 \\ \text { RMSD bond lengths }(\AA) & 0.009 & 0.008 \\ \text { RMSD bond angles }\left({ }^{\circ}\right) & 1.4 & 1.3 \\ \text { Average B-factors }\left(\AA^{2}\right) & & \\ \text { With TLS contribution } & 54.5 & 27.6(42.1) \\ \text { Ramachandran plot } & & \\ \text { Preferred regions }(\%) & 94.8 & 97.0 \\ \text { Allowed regions }(\%) & 4.4 & 2.5 \\ \text { Outliers (\%) } & 0.9 & 0.6\end{array}$

$\mathrm{X}$-ray diffraction data were collected on one crystal. Values in parentheses are for highest-resolution shell. 

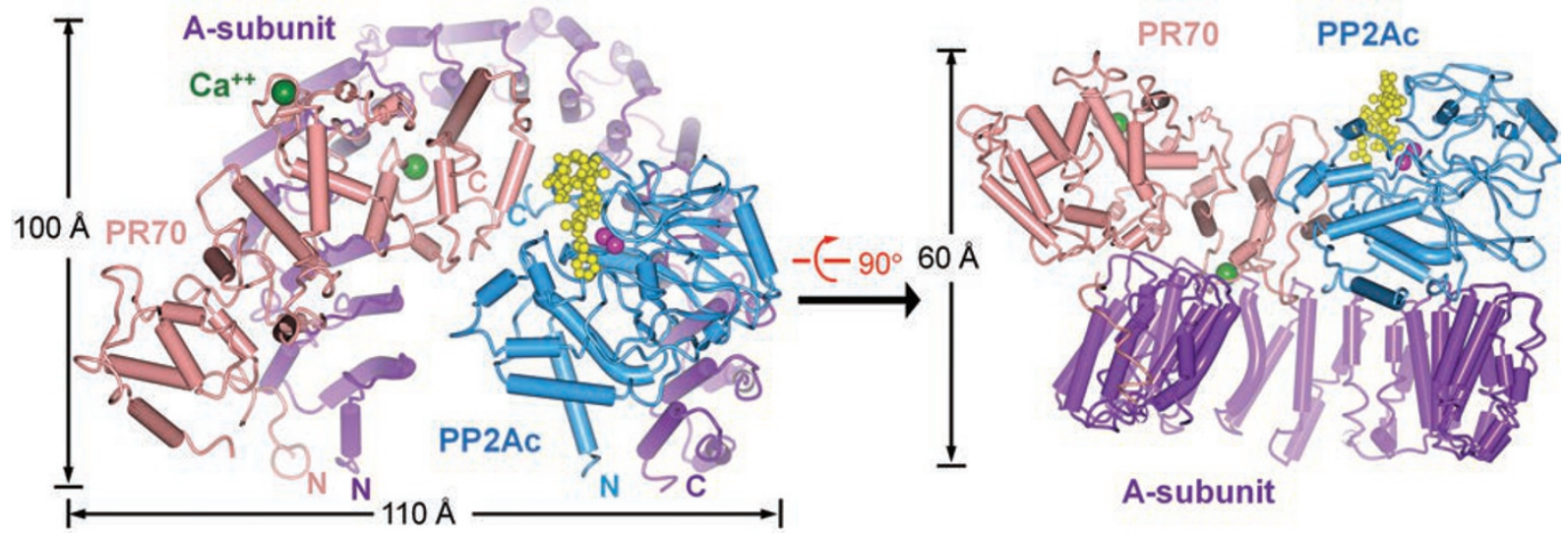

A-subunit

Figure 1 Overall structure of the heterotrimeric PP2A holoenzyme containing PR70 bound to MCLR. Two perpendicular views are shown. The scaffold (A-subunit), catalytic (PP2Ac) and regulatory B" (PR70) subunits are shown in worm and tube and colored purple, cyan and orange, respectively. MCLR is shown in yellow sphere. See also Supplementary information, Figure S1.

PR61 families of PP2A regulatory subunits [22-24]. In the holoenzyme containing PR70, the catalytic subunit associates with the $\mathrm{C}$-terminal five HEAT repeats, and PR70 binds to HEAT repeats 1-7 at the N-terminus of the A subunit (Figure 1). The PR70 binding region overlaps with the binding sites of the B/PR55 and B'/PR61 regulatory subunits. This supports the mutually exclusive binding of different B subunits to the heterodimeric core enzyme of PP2A.

The A subunit in the PR70 holoenzyme adopts a distinctly compact conformation compared with other PP2A complexes. The compact conformation of the A subunit in the PR70 complex shortens the width but increases the height of the A subunit, and thus increases the overall height of the PR70 holoenzyme. A helix domain following the N-terminal hydrophobic motif of PR70 extends outside the rim of the A subunit, resulting in a holoenzyme that is wider than the B/PR55 and B'/B56 holoenzymes. Consistent with this structural observation, although PR70 (108-519) alone migrated as a smaller protein than the $\mathrm{B}^{\prime} \varepsilon$ regulatory subunit during gel filtration, the holoenzyme containing PR70 (108-519) migrated as a slightly larger complex than the holoenzyme containing the $\mathrm{B}^{\prime} \varepsilon$ regulatory subunit (Supplementary information, Figure S1).

The PR70 subunit is an elongated protein harboring an $\mathrm{N}$-terminal hydrophobic motif and two EF hand calciumbinding motifs that bind to the PP2A core enzyme with both scaffold and catalytic subunits (Figures 1 and 2). The N-terminal hydrophobic motif (125-129) and a broad surface in the protein core encompassing EF2 directly contacts the A subunit. Interestingly, a helix (439-
446) of PR70 interacts directly with the catalytic subunit near the active site, which might play an important role in substrate orientation.

\section{Comparison between PR70 and PR72}

Comparison of the primary sequences of the B"/PR72 family subunits showed that although they have unique $\mathrm{N}$ - and C-terminal regions, each member of the family contains a conserved central region encompassing the N-terminal hydrophobic motif and the two EF hand calcium-binding motifs, including the helix that binds directly to the catalytic subunit (Figure 2A). This suggests that all B"/PR72 subunits might associate with the core enzyme in a similar manner, and share similar regulation by calcium binding.

Two structures of PR72 were determined: one with both $\mathrm{EF}$ hands associated with $\mathrm{Ca}^{2+}$ ions and another in which only the second EF hand (EF2) is associated with $\mathrm{Ca}^{2+}$ (Figure 2B). This is consistent with the previous observation that EF2 has a higher binding affinity for $\mathrm{Ca}^{2+}$ than EF1 [14]. Consistent with their sequence similarity, overlaying the structures of the isolated PR72 subunit and the PR70 from the holoenzyme shows that they share a similar structural core encompassing the two EF hand calcium-binding motifs, with a root-mean-squaredeviation of $1.44 \AA$ over 262 residues (Figure 2B). These results suggest that members in the $B^{\prime \prime} / P R 72$ family have similar structures, and assembly into the heterotrimeric holoenzyme does not induce much of an overall change in conformation of the protein core of the PR70/72 subunits. 
A

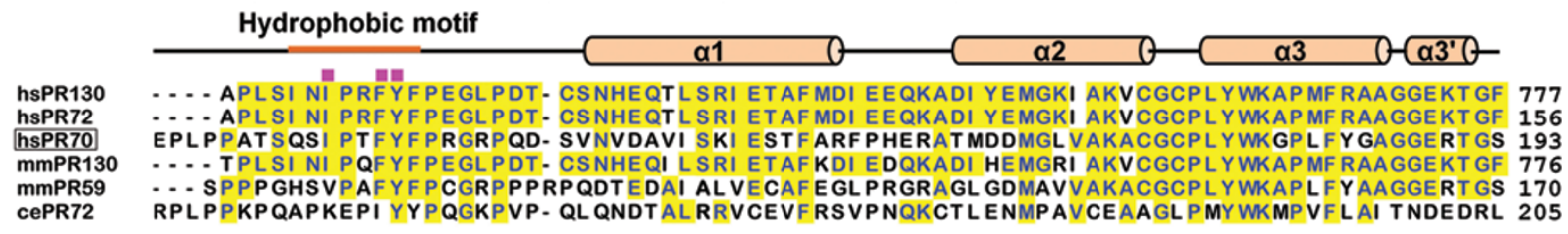

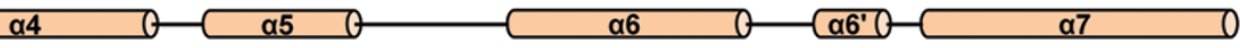

hsPR130 VTAQSFI AMWRKLLNNHHDDASKFI CLLAKPNCSSLEQEDFI PLLQDVVDTHPGLTFLKDAPEFHSRYI TTVI QRI FYTV 857 hSPR72 VTAQSFI AMWRKLLNNHHDDASKFI CLLAKPNCSSLEQEDFI PLLQDVVDTHPGLTFLKDAPEFHSRYI TTVI QRI FYTV 236 hSPR70 VSVHKFVAMWRKI LQNCHDDAAKFVHLLMSPGCNYLVQEDFVPFLQDVVNTHPGLSFLKEASEFHSRYI TTVI QRI FYAV 273 mmPR130 VTAQSFI AMWKKLLSNRHDDAAKFI W. LAKPSCSYLEQEDFI PLLQDVVDTHPGLTFLKDAPEFHSRYI TTVVQRI FYTV 856 mmPR59 VSVHMFVAMWRKVLLTCHDDAARFVRLLGHPGCSGLI QEDFVPFLQDVVNSHPGLAFLRAAKDFHSRYI TTVI QRI FYTV 250 CEPR72 ATQVDFTSWWKAMT SVAHDEAARFVYTLTMGSRSYLQPEDFYEML MDVI HT HPGLAFYRDATEF HDKY CQVVMT RI F WND 285

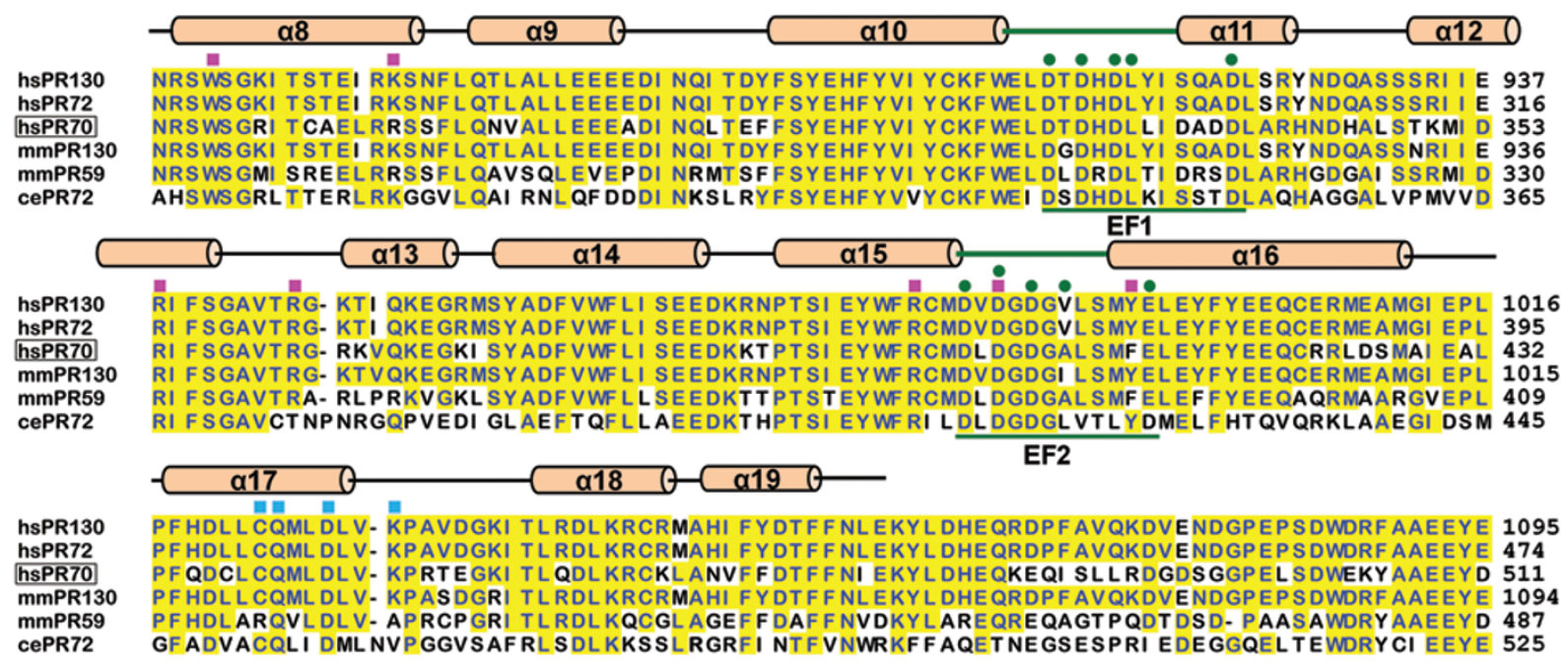

- Scaffold subunit binding $\quad \because$ Catalytic subunit binding $\quad \bullet$ Calcium ion chelation

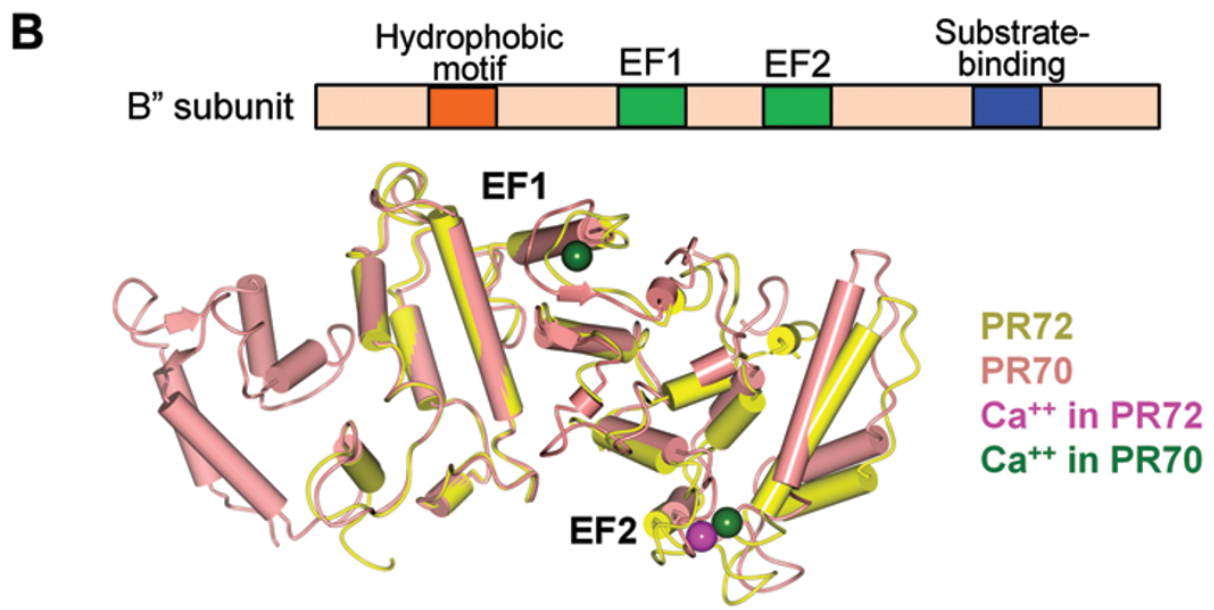

Figure 2 Sequence and structural comparison of B" regulatory subunits. (A) Sequence alignment of B" regulatory subunits from human (hs), mouse ( $\mathrm{mm}$ ) and C. elegans (ce). Secondary structural elements are indicated above the sequences. Conserved residues are highlighted in yellow. Residues that interact with the scaffold and catalytic subunits are identified by magenta and cyan squares, respectively. The residues that are involved in calcium binding are indicated by green circles. The EF-hand calcium-binding motifs are underlined by green lines. (B) Illustration of the functional domains of B" subunit (upper panel) and overlay of the structures of PR72 in isolation (yellow) and PR70 from the holoenzyme (orange) (lower panel). The PR72 subunit with the mono-calcium bound form is shown. The hydrophobic motif is missing in PR72 core. 
Interfaces of PR70 with the scaffold and catalytic subunits

The interaction between the PR70 regulatory subunit and the PP2A core enzyme can be best described by three separate interfaces, two at the interface with the A subunit and one at the interface with the catalytic subunit (Figure 3A). At the first interface with the A subunit (AB-1), the N-terminal hydrophobic motif of PR70 latches to a hydrophobic surface on the side of the first HEAT repeat via PR70 residues Ile125 and Phe128 (Figure $3 \mathrm{~B})$. The second interface $(\mathrm{AB}-2)$ harbors extensive contacts between the PR70 protein core and the top ridge of HEAT repeats 2-7 that are enriched in hydrogen bond (H-bond), salt bridge, and stacking interactions (Figure 3C). In particular, two acidic residues in HEAT repeats 2 and 3, Asp61 and Glu100, make H-bond and salt bridge contacts to Arg288 and Arg398 of PR70. Two basic residues in repeats 5 and 7, Arg183 and Arg258, make Hbond and salt bridge interactions to Asp403 in PR70. The $\pi-\pi$ stacking interactions are found in the middle of the interface, formed between Trp140 in HEAT repeat 4 and PR70 residues, Trp277, Arg362, and Arg398. Note that Asp403 also participates in the chelation of $\mathrm{Ca}^{2+}$ ions by EF2 of PR70. This architecture places the EF2 calciumbinding motif in direct contact with the A subunit of PP2A. Consistent with this structural observation, depletion of calcium from PR70 by EDTA abolished the association between PR70 and the PP2A core enzyme (Figure $3 \mathrm{D})$, reflecting the critical importance of EF2 in the assembly of PP2A holoenzymes with B"/PR72 regulatory subunits $[6,14,25]$.

PR70 interacts with the catalytic subunit near the phosphatase active site with a direct contact with the $\beta 12-\beta 13$ loop that hangs over the active site (Figure $3 \mathrm{E}, \mathrm{BC}$ interface). A PR70 helix (residues 439-446) is nestled into a shallow groove of the catalytic subunit, formed by Tyr91, Tyr267 (on the $\beta 12-\beta 13$ loop), Arg294, and Arg295. These residues make extensive H-bond and salt bridge interactions with PR70 residues Cys439, Gln440, Asp443, and Lys446. The PP2Ac-binding helix is located at the beginning of the C-terminal sequence (residues 440-575) of PR70, which was previously suggested to contain an essential Cdc6-binding region [13, 14]. Because the PP2Ac-binding helix is buried in the holoenzyme and not available for interaction with Cdc6 (Figure 3A, 3E), this Cdc6-binding region is most likely located after residue 446 . It might be closely linked to the PP2Ac-binding helix, and thus expected to be in a close proximity to the phosphatase active site in the holoenzyme (Figure 3E). This architecture suggests that the interaction between PR70 and the catalytic subunit might play an important role in orienting Cdc6 for dephospho- rylation.

Collectively, the interactions between PR70 and the PP2A core enzyme bury a solvent accessible surface area of $3200 \AA^{2}$ and $1300 \AA^{2}$ at the interfaces with the $A$ and the catalytic subunits, respectively. As already mentioned, given that the PR70 residues at the interface with the core enzyme are well-conserved in the B"/PR72 family (Figure 2A), we expect that other B"/PR72 regulatory subunits may have similar contacts with the PP2A core enzyme, and argue that the structure of PR70 holoenzyme can serve as a representative model of all the holoenzymes in this family.

\section{Substrate recognition and holoenzyme conformation}

The structures of trimeric PP2A holoenzymes containing $\mathrm{B} \alpha / \mathrm{PR} 55 \alpha, \mathrm{B}^{\prime} \gamma 1 / \mathrm{PR} 61 \gamma 1$ [22-24], and PR70 regulatory subunits allowed us to compare the three holoenzymes. As expected from their divergent sequences, the three families of regulatory subunits share no structural similarity. The B/PR55 regulatory subunits contain structural repeats that form seven-bladed $\beta$ propeller and the B'/PR61 subunits contain eight HEAT repeats. The putative substrate-binding site of these regulatory subunits is located on their top surfaces, involving several of their structural repeats. In contrast, the B"/PR72 subunit has a linear arrangement of different functional motifs with the Cdc6-binding region located at the C-terminus (after residue 446). Due to a direct contact between PR70 and the catalytic subunit near the active site (Figure 3E), the putative Cdc6-binding region is expected to be near the phosphatase active site (Figure 4A). We thus speculate that the B"/PR72 subunits likely have a mechanism for orienting substrates different from the B/PR55 and $\mathrm{B}^{\prime} /$ PR61 regulatory subunits.

A notable difference between the three families of PP2A holoenzymes is their distinct A subunit conformations (Figure 4B). The A subunit undergoes a drastic conformational change in all three holoenzymes, pivoting around a hinge region between HEAT repeats 10 and 11 [21-24]. The A subunit in the holoenzyme containing the $\mathrm{B}^{\prime} \gamma 1$ regulatory subunit has a more compact conformation than the holoenzyme containing the $\mathrm{B} \alpha$ subunit, likely due to the extensive interaction of $\mathrm{B}^{\prime} \gamma 1$ with the catalytic subunit $[23,24]$. The A subunit in the PR70 holoenzyme is even more compact. The measured distances between the $\mathrm{N}$ - and $\mathrm{C}$-termini of the A subunit were $70 \AA$, $53 \AA$, and $34 \AA$ in the $\mathrm{B} \alpha, \mathrm{B}^{\prime} \gamma 1$, and PR70 holoenzymes, respectively. The tripartite binding sites of PR70 likely lock the A subunit into this compact conformation. These structural observations show that the conformational dynamics of the scaffold subunit is required for the assembly of diverse PP2A holoenzymes, and underlie an 

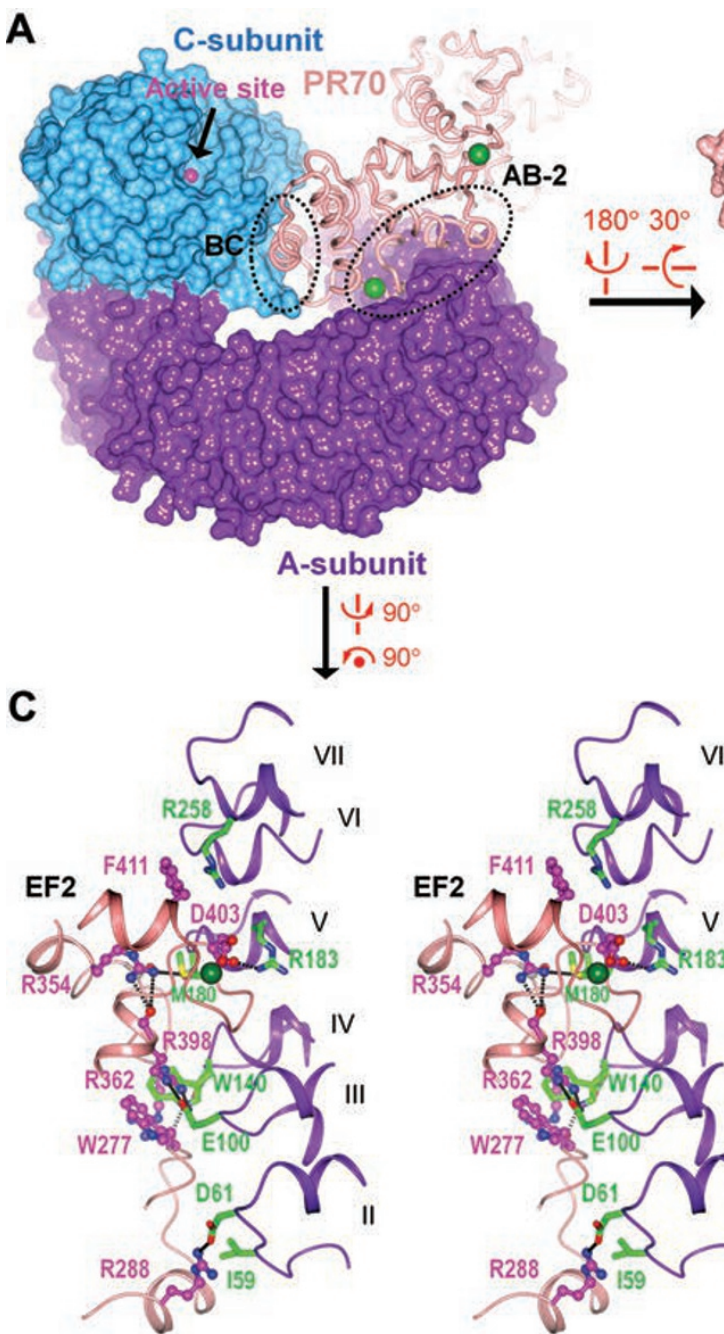<smiles>C1CCCC1</smiles>
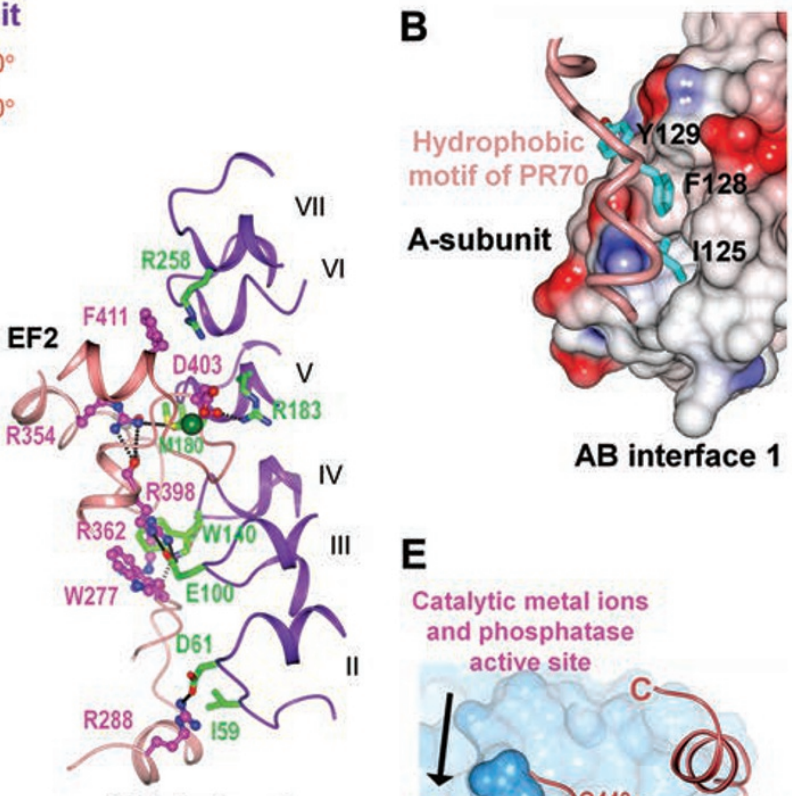

$A B$ interface 1

\section{E}

Catalytic metal ions and phosphatase active site

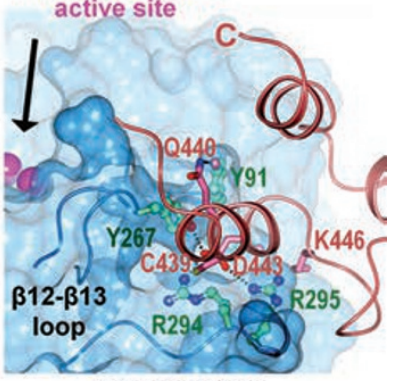

$B C$ interface

D

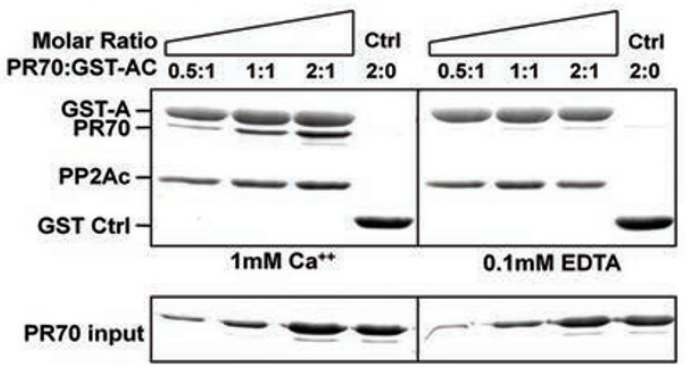

Figure 3 Intersubunit interfaces in the trimeric PP2A holoenzyme containing PR70. The color scheme is the same as Figure 1. (A) Two opposite views of the PR70 holoenzyme illustrate three separated interfaces to the $A$ subunit (AB-1, $A B-2)$ and the catalytic subunit $(B C)$. The structures are shown in the surface or worm. (B) A close-up view of the AB-1 interface between the protruding N-terminal hydrophobic motif of PR70 and a hydrophobic surface of the A subunit HEAT repeat 1 (electrostatic potential is shown). (C) A close-up stereo of the AB-2 interface between an extensive surface of PR70 encompassing the second EF hand motif (EF2) and the A subunit HEAT repeats 2-7 (II-VII). Residues of PR70 and the A subunit are shown in ball-and-stick and cylinder, and colored magenta and green, respectively. $\mathrm{H}$-bond is indicated by dashed black lines. (D) Pull down of titrated concentrations of PR70 with bound and depleted calcium, respectively, by GST-tagged PP2A core enzyme (GST-A bound to PP2Ac). Experiments were repeated three times; representative results are shown. (E) A close-up view of the interface between PR70 and the catalytic subunit (BC interface). Residues of PR70 and the catalytic subunit are shown in cylinder and ball-and-stick, and colored orange and green, respectively. H-bond is indicated by dashed black lines. The catalytic metal ions are shown in sphere and colored magenta. 
A

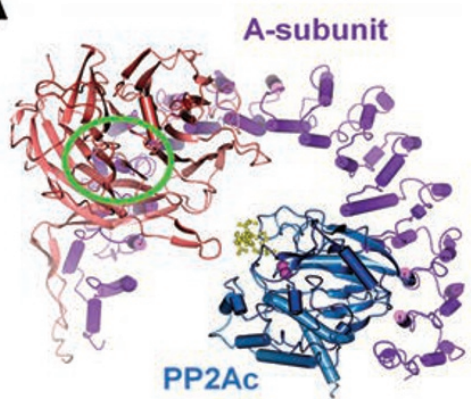

PP2A holoenzyme containing $\mathrm{B} / \mathrm{B} 55$ subunit
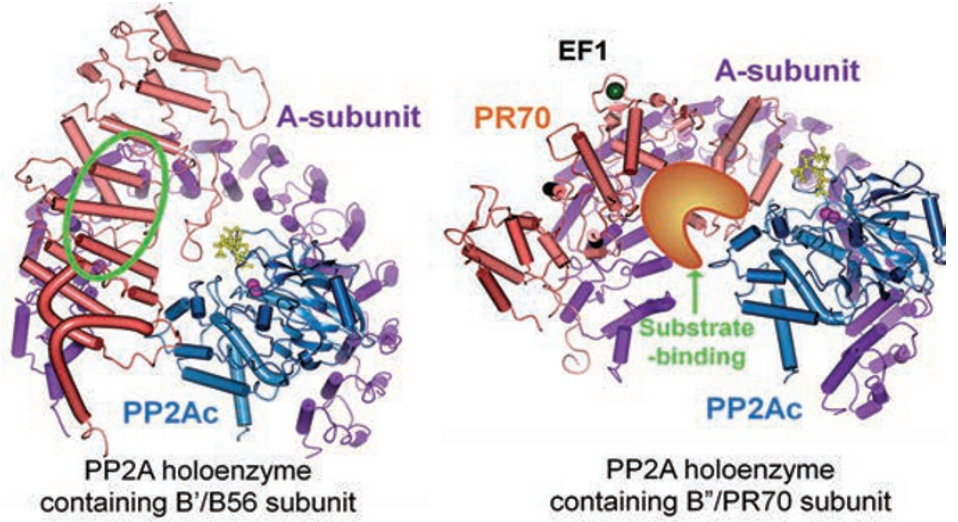

PP2A holoenzyme containing B"/PR70 subunit

B

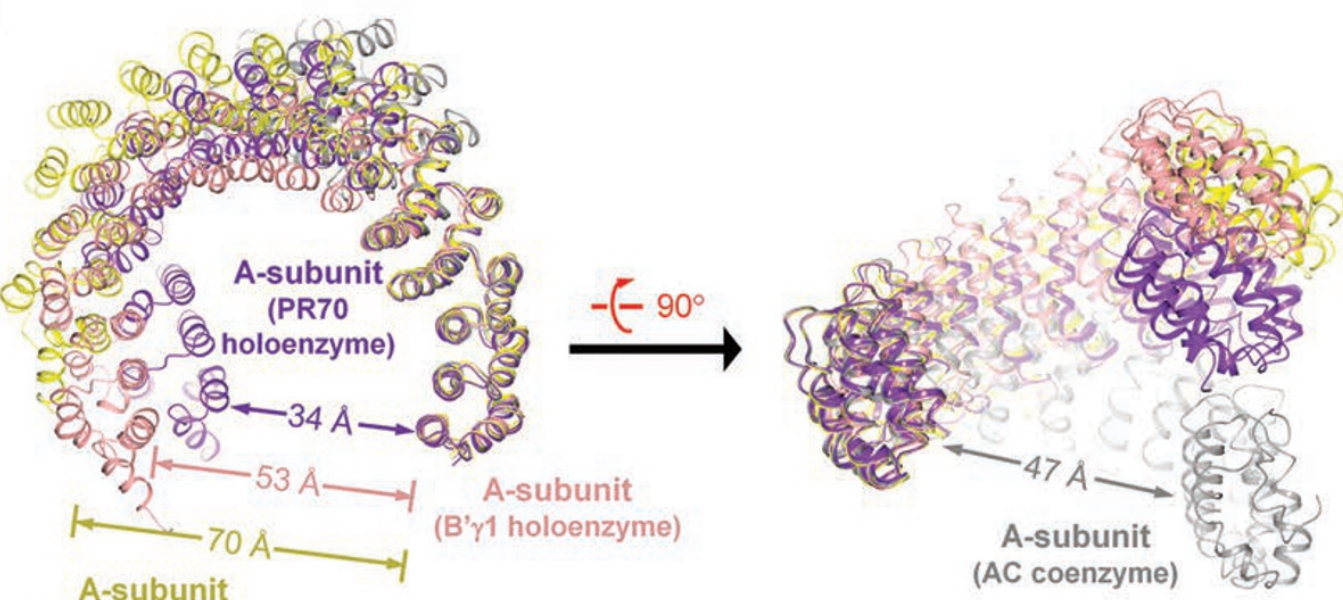

(Ba holoenzyme)

C

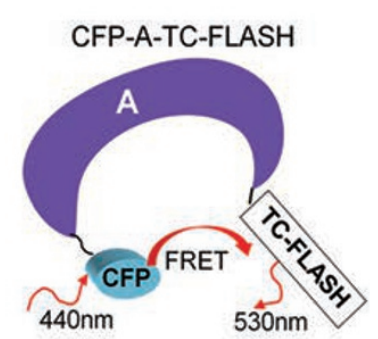

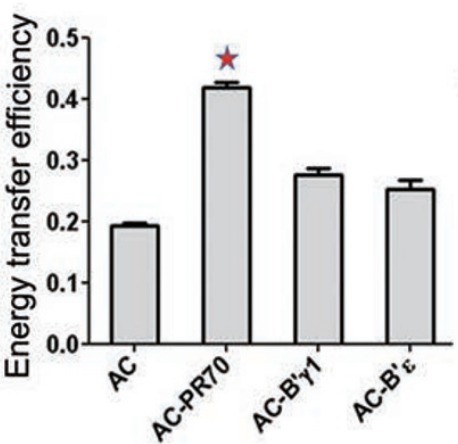

Input for FRET assay

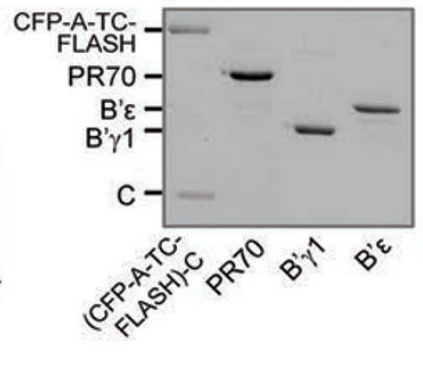

Figure 4 Insights into substrate binding and holoenzyme conformation of the PR70 holoenzyme in comparison with holoenzymes containing the B/PR55 and B'/PR61 family of regulatory subunits. (A) Structures of PP2A holoenzymes containing B/ PR55 (left, PDB code: 3DW8), B'/PR61 (middle, PDB code: 2NPP) and B"/PR70 (right), respectively. Green spherical circles indicate the putative substrate-binding sites on the top surface of the holoenzymes containing B/PR55 and B'/PR61 subunits spanning several structural repeats of the regulatory subunits (left and middle). The predicted position of the previously suggested substrate-binding site of PR70 and the top surface location of EF1 are indicated (right). (B) Conformational variation of the A subunit in PP2A core enzyme and holoenzymes containing B $\alpha, B^{\prime} \gamma 1$ and PR70 manifested by structural overlay via the catalytic subunit and the C-terminal five HEAT repeats of the A subunit. The A subunits from core enzyme and holoenzymes containing $B \alpha, B^{\prime} \gamma 1$ and PR70 are colored grey, yellow, orange and purple, respectively, and the distances between their $\mathrm{N}$ - and $\mathrm{C}$-termini are shown. (C) FRET assay measured changes of the distance between the $\mathrm{N}$ - and $\mathrm{C}$-termini of the A subunit between the core enzyme and the holoenzymes containing B' and PR70 (108-575) subunits. The scheme for FRET is shown (left) and the protein input for the FRET assay was examined by SDS-PAGE (right). 
A

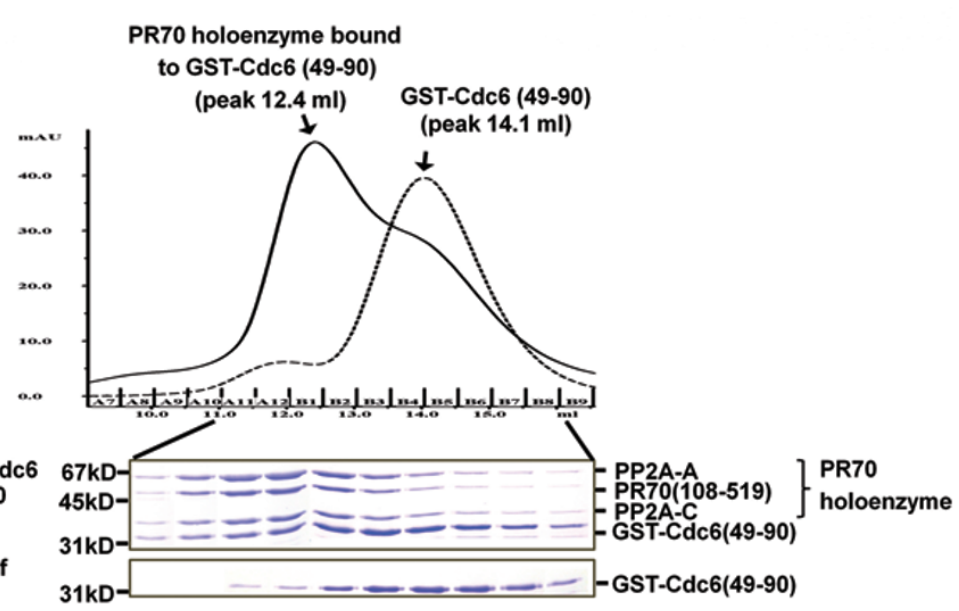

B

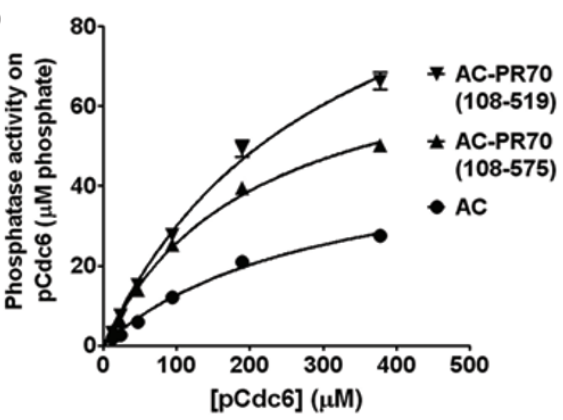

\begin{tabular}{|c|c|c|c|}
\hline $\begin{array}{c}\text { pCdc6 as } \\
\text { substrate }\end{array}$ & AC & $\begin{array}{c}\text { AC-PR70 } \\
(108-575)\end{array}$ & $\begin{array}{c}\text { AC-PR70 } \\
(108-519)\end{array}$ \\
\hline $\begin{array}{c}\mathrm{K}_{\text {cat }} \\
\left(\mathrm{min}^{-1}\right)\end{array}$ & $15.8 \pm 1.3$ & $24.7 \pm 1.3$ & $38.3 \pm 2.9$ \\
\hline $\mathrm{K}_{\mathrm{m}}(\mu \mathrm{M})$ & $299 \pm 46$ & $208 \pm 22$ & $308 \pm 42$ \\
\hline $\begin{array}{c}\mathrm{K}_{\text {cat }} / \mathrm{K}_{\mathrm{m}}(\mu \mathrm{M}- \\
\left.1 \mathrm{~min}^{-1}\right)\end{array}$ & 0.05 & 0.12 & 0.12 \\
\hline
\end{tabular}

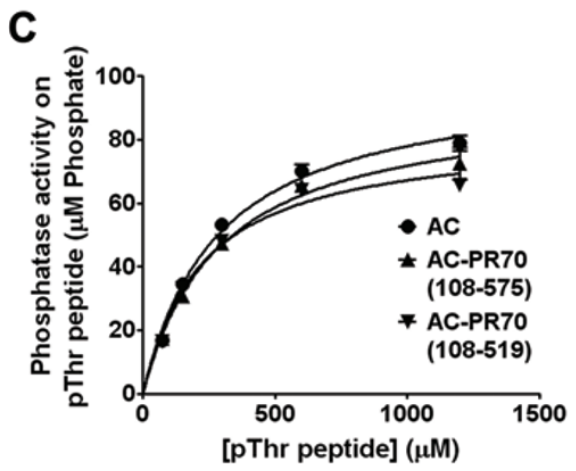

\begin{tabular}{|c|c|c|c|}
\hline $\begin{array}{c}\mathrm{pThr} \\
\text { peptide as } \\
\text { substrate }\end{array}$ & AC & $\begin{array}{c}\text { AC-PR70 } \\
(108-575)\end{array}$ & $\begin{array}{c}\text { AC-PR70 } \\
(108-519)\end{array}$ \\
\hline $\begin{array}{c}\mathrm{K}_{\text {cat }} \\
\left(\mathrm{min}^{-1}\right)\end{array}$ & $1254 \pm 51$ & $1158 \pm 53$ & $1028 \pm 48$ \\
\hline $\mathrm{K}_{\mathrm{m}}(\mu \mathrm{M})$ & $286 \pm 35$ & $291 \pm 38$ & $225 \pm 32$ \\
\hline $\begin{array}{c}\mathrm{K}_{\mathrm{cat}} / \mathrm{K}_{\mathrm{m}} \\
\left(\mu \mathrm{M}^{-1} \mathrm{~min}^{-1}\right)\end{array}$ & 4.4 & 4.0 & 4.6 \\
\hline
\end{tabular}

Figure 5 The PR70 holoenzyme binds to Cdc6 and specifically enhances Cdc6 dephosphorylation. (A) The PR70 holoenzyme and GST-Cdc6 (49-90) co migrated over gel filtration chromatography. Protein fractions were examined by SDS-PAGE and visualized by Coomassie blue staining. (B) Steady state enzyme kinetics of PP2A core enzyme and the holoenzymes containing PR70 (108-519) or PR70 (108-575) (0.16 $\mu \mathrm{M})$ toward pCdc6 (49-90) (12-378 $\mu \mathrm{M})$. (C) Steady state enzyme kinetics of PP2A core enzyme and the holoenzymes containing PR70 (108-519) or PR70 (108-575) (0.4 nM) toward pThr peptide (75-1 $200 \mu \mathrm{M})$. All experiments were repeated three times. For (B and $\mathbf{C}$ ), the normalized enzyme input for the assays were shown in Supplementary information, Figure S2. The experiments were performed in triplicates and the calculated $\mathrm{K}_{\mathrm{cat}}$ and $\mathrm{K}_{\mathrm{m}}$ and the $\mathrm{K}_{\text {cat }} / \mathrm{K}_{\mathrm{m}}$ ratio are summarized in table on the right in each panel.

important basis for regulating holoenzyme assembly and function.

To probe the conformational differences caused by different PP2A regulatory subunits, we established a FRET (fluorescence resonance energy transfer) assay, in which the A subunit is fused to CFP (cyan fluorescent protein) at its $\mathrm{N}$-terminus and a tetracysteine peptide (TC) at its C-terminus. Covalent labeling of the TC peptide by the FlAsH-EDC ${ }_{2}$ compound [26] genetates a highly fluorescent fluorophore (TC-FLASH) that serves as an acceptor for CFP in FRET [27, 28] (Figure 4C, left). The A subunit harboring the fluorophore pair was then used to 


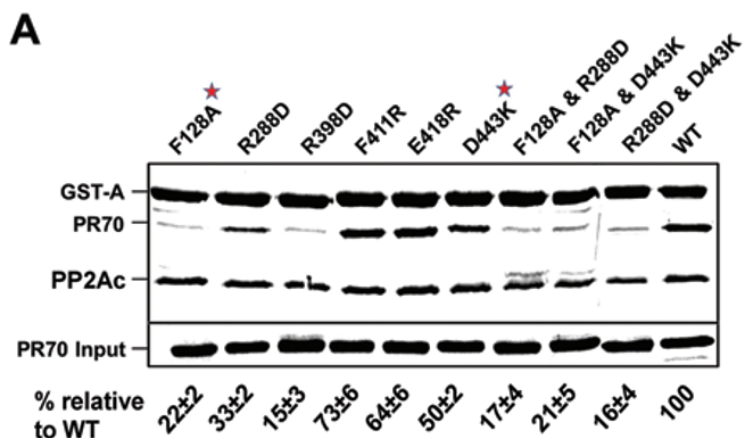

C

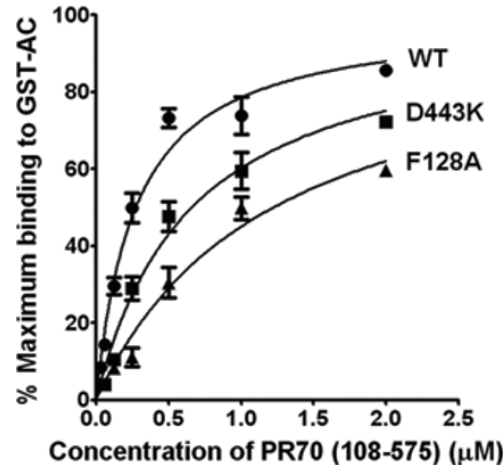

D
B

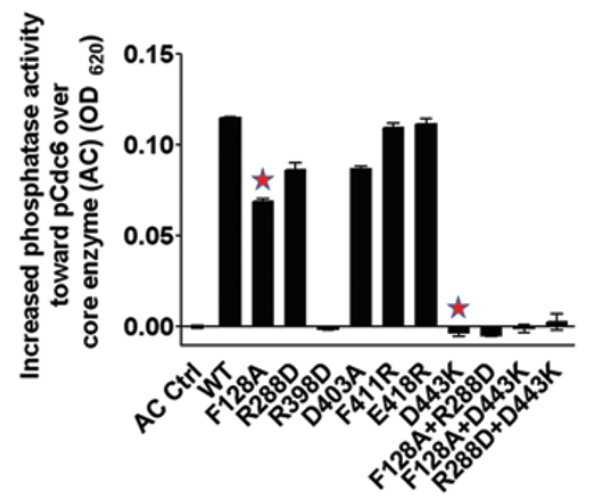

PP2A holoenzymes with PR70 mutants

\begin{tabular}{|c|c|c|c|}
\hline PR70 (108-575) & WT & F128A & D443K \\
\hline $\begin{array}{c}\text { Estimated binding affinity } \\
\text { to GST-AC (Kd ( }(\mu \mathrm{M}))\end{array}$ & 0.3 & 1.2 & 0.7 \\
\hline $\begin{array}{c}\text { (Figure 6C) } \\
\text { Effect on binding affinity } \\
\text { (fold of changes) }\end{array}$ & 1 & $\downarrow 4$ & $\downarrow 2$ \\
\hline $\begin{array}{c}\text { Enhanced phosphatase } \\
\text { activity toward pCdc6 } \\
\text { (Figure 6B) }\end{array}$ & $100 \%$ & $60 \%$ & $0 \%$ \\
\hline
\end{tabular}

Figure 6 Mutational analysis of the tripartite PR70 interfaces in holoenzyme assembly and enhancement of phosphatase activity toward pCdc6. (A) In vitro pull down of wild-type and mutant PR70 (108-575) proteins by GST-AC (PP2A core enzyme). Experiments were repeated three times; representative results are shown. The normalized percentage of binding was averaged from three experiments; mean \pm SEM was shown below the data. (B) The increased phosphatase activity of holoenzymes containing wild-type and mutant PR70 (108-575) proteins compared with the core enzyme toward pCdc6. The phosphatase activity was measured using $200 \mathrm{nM}$ of PP2A and $60 \mu \mathrm{M}$ of pCdc6 substrate. Experiments were performed in triplicate and repeated three times; representative results are shown. For both panels, red stars indicate two mutations, F128A and D443K, that exhibited distinctively different effect on holoenzyme assembly and phosphatase activity toward pCdc6. These mutations involves the $\mathrm{N}$-terminal hydrophobic (AB-1) interface with the A subunit and the interface with the catalytic subunit (BC) (Figure 3), respectively. (C) Simulation of concentration-dependent binding of PR70 (108-575) wild-type and mutants to GST-AC based on data shown in (Supplementary information, Figure S3C for estimation of binding affinity (Kd). (D) Summary of the effect of PR70 mutations on the binding affinity between PR70 and the core enzyme and on enhancement of phosphatase activity toward Cdc6. See also Supplementary information, Figure S3.

assemble PP2A complexes, and the efficiency of energy transfer was determined. As expected, the energy transfer efficiency of the PR70 holoenzyme is significantly higher than the core enzyme and the holoenzyme containing B'/ PR61 family regulatory subunits (Figure 4C, middle). This is consistent with the structural observations discussed above (Figure 4B).

In vitro Cdc6 dephosphorylation by the PR70 holoenzyme

The C-terminal sequence of PR70 (440-575) was previously shown to harbor a binding region for the $\mathrm{N}$ terminal fragment of Cdc6, and this interaction is needed for the association of Cdc6 with PP2A scaffold and cata- lytic subunits in cells $[13,14]$. To extend this observation, we showed that GST-Cdc6 (49-90), a GST-tagged Cdc6 fragment containing residues 49-90, comigrated readily well with the PP2A holoenzyme containing PR70 (108-519) (Figure 5A). Together with the structural insights mentioned earlier, these results suggest that this Cdc6-binding region is likely located within the PR70 sequence containing residues 447-519. The specific interaction between Cdc6 and the PR70 holoenzyme suggests that this holoenzyme might target $\mathrm{Cdc} 6$ for dephosphorylation.

To test this notion, we established an in vitro phosphatase assay toward phosphorylated Cdc6. Purified GSTCdc6 (49-90) was phosphorylated in vitro using cyclin A/ 
CDK2, and the GST-tag was removed. The phosphorylated Cdc6 peptide (pCdc6) was then isolated by gel filtration, and used as the substrate for measuring the phosphatase activity of the PP2A core enzyme and the holoenzymes containing PR70. Two different fragments of PR70 (108-519, and 108-575) were tested to determine the effect of the C-terminal PR70 sequence on the holoenzyme activity toward Cdc6. At the same molar concentration, the trimeric holoenzymes containing either of the two PR70 fragments exhibited enhanced phosphatase activity toward pCdc6 compared with the heterodimeric core enzyme (Figure $5 \mathrm{~B}$ ). The calculated $\mathrm{K}_{\mathrm{cat}} / \mathrm{K}_{\mathrm{m}}$ ratio is almost identical for both PR70 holoenzymes, which is significantly higher than the core enzyme (AC) (Figure $5 \mathrm{~B}$, right). In contrast, both PR70 holoenzymes and the core enzyme were equally active toward a synthetic pThr peptide (K-R-pT-I-R-R), a universal substrate for all PP2A complexes (Figure 5C). These data support the notion that the PR70 subunit enhances the phosphatase activity of PP2A toward pCdc6.

Tripartite PR70 interfaces in holoenzyme assembly and Cdc6 dephosphorylation

To examine whether the multiple binding sites of PR70 are essential for holoenzyme structure and function, we performed mutational analysis at all three in- terfaces. Mutations at both of the interfaces with the A subunit, $\mathrm{AB}-1$ and $\mathrm{AB}-2$, particularly F128A and R398D, severely compromised the interaction between PR70 and the GST-tagged PP2A core enzyme (Figure 6A). This result is consistent with the previous observation that the hydrophobic motif of PR70 is required for holoenzyme assembly in cells [14]. The PR70 mutation at the interface with the catalytic subunit, D443K, exhibited a less severe defect on holoenzyme assembly, and reduced the interaction between PR70 and the GST-tagged PP2A core enzyme only by half. None of these mutations affected the interaction between PR70 and Cdc6 (Supplementary information, Figure S3A).

Next, we investigated how PR70 mutations affect the activity of PR70 to enhance phosphatase activity toward pCdc6. Mutation of PR70 at the interface with the catalytic subunit, D443K, which retained considerable activity in holoenzyme assembly (Figure 6A), completely abolished the ability of PR70 to enhance phosphatase activity toward pCdc6 (Figure 6B). In contrast, this mutation barely affected the holoenzyme activity toward the pThr peptide (Supplementary information, Figure S3B), indicating that it did not affect the phosphatase active site. Unlike D443K, the F128A mutation had a much smaller defect in enhancing phosphatase activity toward pCdc6 (Figure 6B), albeit it had a more severe defect in
A

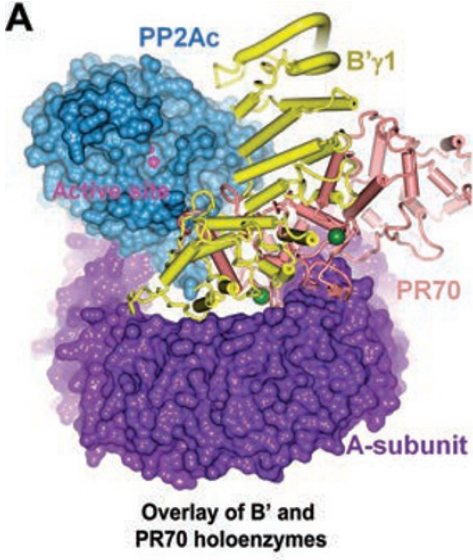

B

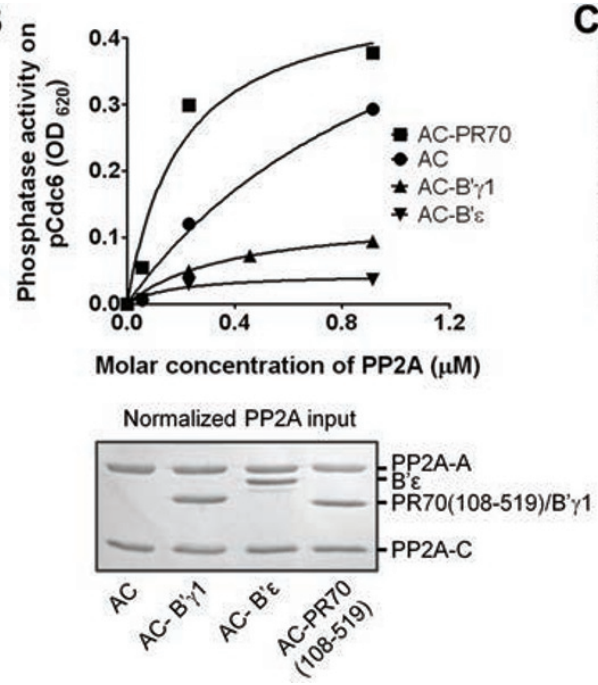

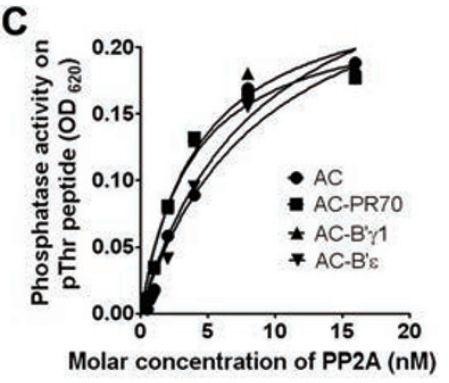

Molar concentration of PP2A (nM)

Figure 7 Restriction of phosphatase activity toward pCdc6 by $B^{\prime}$ regulatory subunits. (A) Overlay of the structure of the $B^{\prime} \gamma 1$ holoenzyme (PDB code: 2NPP) to the PR70 holoenzyme via the catalytic subunit and the C-terminal five HEAT repeats of the A subunit. The structural alignment illustrates different binding sites of $B^{\prime} \gamma 1$ and PR70 on the catalytic subunit near the phosphatase active site. (B) PP2A concentration-dependent dephosphorylation of pCdc6 (60 $\mu \mathrm{M})$ by PP2A core enzyme and holoenzymes containing PR70 (108-519), B' $\gamma 1$, or B' $\varepsilon$ subunits. Normalized PP2A input for the assay was examined by SDS-PAGE and visualized by Coomassie blue staining (lower panel) (C) PP2A concentration-dependent dephosphorylation of pThr peptide $(100 \mu \mathrm{M})$. The PP2A complexes tested and normalization of PP2A input were the same as in B. See also Supplementary information, Figure S4. 
holoenzyme assembly (Figure 6A).

To better define the different effect of PR70 D443K and F128A mutations on holoenzyme assembly and enhancement of PP2A activity toward pCdc6, we estimated the binding affinity between PP2A core enzyme and PR70 using a titration pull-down assay to determine the effect of these two mutations on holoenzyme assembly (Supplementary information, Figure S3C, Figure 6C). The F128A mutation was estimated to reduce the binding affinity of PR70 by fourfold, but retained $60 \%$ of the PR70 activity to enhance Cdc6 dephosphorylation. In contrast, the D443K mutation reduced the binding affinity only by twofold, but led to a complete loss of activity to enhance Cdc6 dephosphorylation (Figure 6D). These results demonstrate that the direct contact between PR70 and the catalytic subunit is required for PR70 to enhance PP2A activity toward pCdc6.

Restriction of PP $2 A$ activity toward $p C d c 6$ by the $B^{\prime}$ family of regulatory subunits

Structural overlay of the trimeric PP2A holoenzymes containing $\mathrm{B}^{\prime} \gamma 1$ and $\mathrm{PR} 70$ regulatory subunits shows that the $\mathrm{B}^{\prime}$ and $\mathrm{B}^{\prime \prime}$ families of regulatory subunits have distinctly different contact surface areas on the catalytic subunit (Figure 7A). Furthermore, the electrostatic potential near the active site of the $\mathrm{B}^{\prime} \gamma 1$ holoenzyme is much more acidic than that of either the core enzyme or the PR70 holoenzyme (Supplementary information, Figure S4). The Cdc6 (49-90) fragment that interacts with the PR70 holoenzyme (Figure 5A) contains both positively and negatively charged residues, which matches the alternating pattern of charge distribution near the active site of PR70 holoenzyme (Supplementary information, Figure S4). These structural features suggest that the $\mathrm{B}^{\prime}$ family of regulatory subunits might inhibit the phosphatase activity toward pCdc6 by occupying the entry route of Cdc6 on the catalytic subunit and by repulsive electrostatic interactions. Consistent with these structural analysis, while the trimeric PR70 holoenzyme exhibited an enhanced activity toward $\mathrm{pCdc} 6$, the $\mathrm{B}^{\prime} \gamma 1$ and $\mathrm{B}^{\prime} \varepsilon$ holoenzymes exhibited a markedly reduced phosphatase activity toward pCdc6 compared with the heterodimeric core enzyme (Figure 7B). As a control, all the holoenzymes and core enzyme tested exhibited a similar activity toward the pThr peptide (Figure 7C).

\section{Discussion}

Despite recent advances in PP2A structural biology, many questions remain about the mechanisms underlying PP2A holoenzyme assembly, substrate specificity, and regulation. As the diversity of PP2A holoenzyme functions are dictated by its distinctly different regulatory subunits, a complete understanding of this crucial regulatory enzyme requires structural information about each holoenzyme family. In the studies reported here, we overcame the major hurdle in crystallization of the $\mathrm{B}^{\prime \prime} /$ PR72 family of PP2A holoenzymes and determined the crystal structures of PR72 regulatory subunit in isolation, and the holoenzyme containing the closely related PR70 regulatory subunit.

Our structures provide important insights into the structural basis of the B"/PR72 PP2A family. First, the PR70 and PR72 subunits have a similar structural core and all the residues at the interface with the core enzyme are well conserved, suggesting that members in this family might share a similar holoenzyme structure and regulatory mechanisms. The structure of the PR70 holoenzyme thus can serve as a representative structural model for all members in this family. Second, the PR70 holoenzyme exhibits the most compact scaffold subunit conformation among all known PP2A holoenzyme structures, likely defined by the multiple binding sites between the elongated B"/PR72 regulatory subunits and the PP2A core enzyme. The compact scaffold subunit conformation might contribute to holoenzyme stability in cells and provide a basis for regulating holoenzyme function via modulating scaffold subunit conformation. Third, the two PR70 EF-hand calcium-binding motifs adopt distinct orientations in the PR70 holoenzyme, which provides possible explanations for the effects of calcium binding on holoenzyme function. The first EF hand motif is located on the top surface of the holoenzyme facing the phosphatase active site, suggesting a role of EF1 in regulating substrate binding. This notion is also supported by the effect of EF1 in enhancing the phosphatase activity of the PR72 holoenzyme in response to changes of calcium levels [6]. The EF2 calcium-binding motif directly contacts the scaffold subunit, which explains the critical role of calcium binding in assembly of holoenzymes containing PR72/PR70 subunits [6, 14, 25].

Our work also established an in vitro phosphatase assay that allowed us to examine Cdc6 dephosphorylation by the PR70 holoenzyme. We showed that the PR70 holoenzyme interacts with an N-terminal fragment of Cdc6 containing residues 49-90 in vitro (Figure 5A), and exhibited enhanced phosphatase activity toward pCdc6 (49-90) (Figure 5B). Our study demonstrated tight control of Cdc6 dephosphorylation that likely relies on a continuous route for $\mathrm{Cdc} 6$ entry to the active site formed by PR70 and the catalytic subunit, which is affected by the interaction between two subunits. This notion is supported by several structural and biochemical observations. First, the catalytic subunit-binding helix of PR70 
(residues 439-446) is closely linked to a putative Cdc6binding region (presumably within PR70 sequence 447519 ), and in close proximity to the phosphatase active site (Figure 3E). Second, PR70 mutation at the interface with the catalytic subunit abolished its ability to enhance phosphatase activity toward pCdc6, despite having a modest effect on holoenzyme assembly (Figure 6). It is likely that mutations at this interface alter the orientation of PR70 and the catalytic subunit, and thus distort precise orientation of the substrate at the active site. Finally, this mechanism likely explains the ability of the B'/PR61 regulatory subunits to hinder Cdc6 dephosphorylation, presumably by steric hindrance of Cdc6 docking site on the catalytic subunit. This notion is coherent with the structural observation that the $\mathrm{B}^{\prime}$ and $\mathrm{B}^{\prime \prime}$ families of regulatory subunits have distinctly different contact surface areas on the catalytic subunit (Figure 7A). A similar mechanism was proposed for spinophilin in substrate restriction of protein phosphatase 1 (PP1) [29], a closely related phosphatase in the PPP family. Whether the B' family of regulatory subunits plays a role in restricting the PP2A phosphatase activity toward Cdc6 during cell cycle remains to be determined.

A previous study suggested that the $\mathrm{B}^{\prime}$ family of regulatory subunits control substrate specificity via chargecharge interactions [30]. Indeed, the electrostatic potential near the PP2A active site becomes much more acidic when the core enzyme is associated with the $\mathrm{B}^{\prime} \gamma 1$ regulatory subunit (Supplementary information, Figure S4), which would favor interaction with positively charged residues in the substrate [30]. In contrast, the PR70 holoenzyme exhibits an alternating pattern of positively and negatively charged surface area, which matches the charge distribution of the PR70-binding Cdc6 (49-90) peptide (Supplementary Figure S4). Thus, the interaction between the PR70 holoenzyme and its substrates might be contributed in part by electrostatic potential highly distinct from that of the $\mathrm{B}^{\prime}$ holoenzymes. It is noteworthy that the electrostatic potentials also play an important role in substrate restriction and specificity of PP1 holoenzymes [31, 32]. Collectively, observations from PP2A and PP1 holoenzymes suggest unifying mechanisms of substrate restriction by alteration of electrostatics and/or steric hindrance of substrate binding.

The C-terminal fragment of PR70 (residues 440575) was previously shown to harbor an essential Cdc6binding region $[13,14]$, and the $\mathrm{C}$-terminal boundary of PR70 required for this binding was further refined to residue 519 in this report (Figure 5A). The structure of the PR70 holoenzyme helps further define the boundary of this binding region after residue 446 , as residues 439-446 are buried in the holoenzyme (Figure 3A and
3E) and most likely not involved in Cdc6 binding. It is important to mention that, although the C-terminal sequence harbors a Cdc6 binding region, the role of the N-terminal PR70 sequence prior to residue 439 in Cdc6 binding remained to be determined. The fact that EF1 is required for full phosphatase activity of the PR72 holoenzyme toward DARPP32 in response to changes of calcium level [6] suggests that the N-terminal sequence might participate in substrate binding at least for some members in the B"/PR72 family. Due to the low binding affinity between PR70 and Cdc6, mapping of the PR70binding region of Cdc6 had been challenging. Albeit the current PR70-Cdc6 fusion protein for crystallization of the PR70 holoenzyme did not yield electron density for Cdc6, further attempts with different boundaries for PR70 and Cde6 and crystallization in the absence of phosphatase inhibitor might be helpful for determining the structures of the PR70 holoenzyme bound to Cde6. The lack of electron density for the Cdc6 peptide might be due to its weak interaction with the PR70 holoenzyme and/or steric hindrance caused by phosphatase inhibitor MCLR at the active site. Our studies provide a basis for structural and biochemical studies required for further understanding of substrate recognition and specificity.

Our observations on PP2A substrate restriction by different regulatory subunits likely provide general insights into tight control of PP2A activity for precise control of cellular processes and signaling. The notion of substrate restriction by different PP2A holoenzymes is coherent with the emerging concept on precise control and coordination of PP2A function for cellular signaling [33, 34]. While several families of PP 2 A holoenzymes have been implicated in cell cycle regulation, the ability of the $\mathrm{B}^{\prime} / \mathrm{PR} 61$ regulatory subunits in restricting PP2A activity toward pCdc6 (Figure 7B) suggests a mechanism for tight restriction of PP2A phosphatase activity during cell cycle.

\section{Materials and Methods}

\section{Protein preparation}

All constructs and point mutations were generated using a standard PCR-based cloning strategy. Expression and purification of PP2A A $\alpha$ (9-589), C $\alpha$ (1-309), and PR72 subunits, and assembly of the PP2A core enzyme (A $\alpha-\mathrm{C} \alpha$ heterodimer) followed procedures described previously [21]. Expression and purification of CFP-A $\alpha$ (9-589)-TC fusion protein and the core enzyme containing this protein were similar to A $\alpha$ (9-589). Cdc6 and PR70 with different boundaries were cloned in pQlink vector (Addgene) harboring a GST-tag and a $\mathrm{His}_{8}$-tag, respectively, and a TEV cleavage site between the affinity tag and the protein. The proteins were overexpressed at $23{ }^{\circ} \mathrm{C}$ in $E$. coli strain $\mathrm{DH} 5 \alpha$. The soluble fraction of the E. coli cell lysate was purified over GS4B resin (GE Healthcare) or Ni-NTA resin (Qiagen), and further fractionated 
by anion exchange chromatography (Source 15Q, GE Healthcare) and gel filtration chromatography (Superdex 200, GE Healthcare). PR70, PR72, and their holoenzymes were purified in the presence of $1-2 \mathrm{mM} \mathrm{CaCl}_{2}$.

To facilitate crystallization of PR72 subunit, PR72 was cleaved by $0.2 \mathrm{mg} / \mathrm{ml}$ elastase for $20 \mathrm{~min}$ on ice. The stable protein core was fractionated by anion exchange and gel filtration chromatography to remove peptide fragments. For crystallization of the PR70 holoenzyme, the PP2A core enzyme was fully methylated by PP2A specific methyltransferase (LCMT-1) in the presence of PTPA (PP2A phosphatase activator) at 1:0.5:0.2 molar ratio [33], followed by association with PR70 (122-490) fused to the Cdc6 peptide (70-90) via a flexible linker (STGNASDSSSDSSSSEGDGTV). The sample was fractionated by anion exchange and gel filtration chromatography to remove LCMT-1, PTPA, and the excess amount of PR70. The purified PR70 holoenzyme was mixed with MCLR in a 1:1.2 molar ratio prior to crystallization.

\section{Crystallization and data collection}

Crystals of PR72 protein core were grown at $23{ }^{\circ} \mathrm{C}$ by the hanging-drop vapor-diffusion method by mixing $\sim 8 \mathrm{mg} / \mathrm{ml}$ of protein with an equal volume of a reservoir solution containing $0.1 \mathrm{M}$ $\mathrm{NaH}_{2} \mathrm{PO}_{4} / 0.1 \mathrm{M} \mathrm{K}_{2} \mathrm{HPO}_{4}, 0.1 \mathrm{M}$ MES pH 6.5, and $2 \mathrm{M} \mathrm{NaCl}$. The crystals appeared in $5 \mathrm{~min}$ and grew to full within 3 days. Crystals of the seleno-methionine-substituted PR72 were grown under similar conditions. Crystals were equilibrated in a cryoprotectant buffer containing reservoir buffer with $20 \%$ glycerol (v/v), and flash frozen in a cold nitrogen stream at $-170{ }^{\circ} \mathrm{C}$. Both native and MAD data sets for PR72 were collected at Brookhaven NSLS beamline X29 and processed using the software Denzo and Scalepack [35].

Crystals of the holoenzyme containing the PR70-Cde6 fusion protein bound to MCLR were grown at $18{ }^{\circ} \mathrm{C}$ by the sittingdrop vapor-diffusion method by mixing $\sim 6 \mathrm{mg} / \mathrm{ml}$ of the protein complex with two thirds volume of a reservoir solution containing 7\% PEG3350 (v/v), $0.03 \mathrm{M}$ succinic acid at $\mathrm{pH} 4.75$. The crystals appear in 3 days and grew to full within 2 weeks. Crystals of the holoenzyme with the seleno-methionine-substituted scaffold and PR70 subunits were grown similarly except that the reservoir solution contains $12 \%$ PEG3350 (v/v), $0.1 \mathrm{M}$ sodium malonate $\mathrm{pH}$ 5.0. Crystals were equilibrated in a cryoprotectant buffer and flash frozen as described above. Native and anomalous data sets for the PR70 holoenzyme were collected at APS LS-CAT sector 21 using beamline $\mathrm{F}$ and $\mathrm{D}$, respectively, and processed using the software HKL2000 [35].

\section{Structure determination}

The structure of PR72 in isolation and the holoenzyme containing PR70 were determined by MAD and SAD phasing, respectively, using program CRANK [36] in the CCP4 package [37]. Selenium atoms were located by program AFRO/CRUNCH2 [38] and refined using BP3 [39]. Following phase improvement and density modification, a large fraction of the scaffold and PR70 subunits were automatically built using Buccaneer [40]. Automatic model building for PR72 was performed similarly but used the native data sets. Model errors were corrected manually based on the electron density map followed by manual building of the rest of the model. The structures were built using Coot [41] and refined using REFMAC restraints with TLS [42]. The structures of PR72 bound to one $\mathrm{Ca}^{2+}$ and two $\mathrm{Ca}^{2+}$ ions were refined to $2.1 \AA$ and 2.9
$\AA$, respectively (Table 1 ). The structures of the seleno-methioninelabeled and native PR70 holoenzymes were refined to $2.8 \AA$ and 2.4 $\AA$, respectively (Table 2).

\section{GST-mediated pull down assay}

Approximately $10 \mu \mathrm{g}$ of GST-AC (core enzyme) or GST-Cdc6 was bound to $10 \mu \mathrm{l}$ of glutathione resin via GST tag. The resin was washed with $200 \mu$ lassay buffer three times to remove the excess unbound protein. Then, $10 \mu \mathrm{g}$ or the indicated amount of PR70 constructs, wild type or mutant PR70 (108-575) was added to the resin in a $200 \mu \mathrm{l}$ volume suspended in the assay buffer containing $25 \mathrm{mM}$ Tris ( $\mathrm{pH} \mathrm{8.0)}, 150 \mathrm{mM} \mathrm{NaCl}, 1 \mathrm{mM} \mathrm{CaCl}_{2}$, and $3 \mathrm{mM}$ DTT. The mixture was washed three times with the assay buffer. The proteins remained bound to resin were examined by SDSPAGE, and visualized by Coomassie blue staining. All experiments were repeated three times. For binding of PR70 vacant of calcium, $1 \mathrm{mM} \mathrm{CaCl}_{2}$ was replaced with $0.1 \mathrm{mM}$ EDTA as indicated. For determination of the binding affinity, titration of PR70 (108-575) wild type and mutants $(1 / 32-4 \mu \mathrm{M})$ was added to the immobilized GST-AC for pull down. The immobilized GST was used as control. The level of binding was quantified using Image J, and results from three separate experiments were fitted in GraghPad Prism (GraphPad Software Inc.) after background subtraction to estimate $\mathrm{Kd}$.

\section{FRET assay}

The donor fluorescent signal of CFP of $100 \mu \mathrm{g} / \mathrm{ml}$ of PP2A core enzyme containing CFP-A $\alpha$ (9-589)-TC fusion protein was measured in the presence and absence of FlAsH-EDC ${ }_{2}$ compound (Invitrogen) using a Victor V 1420 Multilabel HTS counter (Wallac) with excitation at $450 \mathrm{~nm}$ and emission at $490 \mathrm{~nm}$. Addition of FlAsH-EDC ${ }_{2}$ compound at 1:1.1 molar ratio creates the highly fluorescent TC-FLASH that serves as the acceptor in the FRET assay. The rate of energy transfer was calculated based on loss of donor fluorescence using the following equation: $E=1-\left(\mathrm{F}_{\mathrm{DA}} / \mathrm{F}_{\mathrm{D}}\right)$, where $\mathrm{F}_{\mathrm{DA}}$ and $\mathrm{F}_{\mathrm{D}}$ are the fluorescence of CFP in the presence and absence of TC-FLASH, respectively. The effect of regulatory subunits on the rate of energy transfer was determined similarly in the presence of five times molar concentration of $\mathrm{B}^{\prime}$ subunits or wild type or mutant PR70 (108-575).

\section{Phosphatase assay}

The purified GST-Cdc6 (49-90) was phosphorylated in vitro by cyclin $\mathrm{A} / \mathrm{CDK} 2(1 / 20 \mathrm{w} / \mathrm{w})$ with $10 \mathrm{mM} \mathrm{MgCl}$ and $10 \times$ molar concentration of ATP for $1 \mathrm{~h}$ at $30{ }^{\circ} \mathrm{C}$. The phosphorylated protein was purified by gel filtration chromatography (Superdex 200, GE Healthcare) to remove free ATP, followed by overnight cleavage of the GST-tag with TEV protease $(1 / 20 \mathrm{w} / \mathrm{w})$. The pCdc6 peptide was then separated from GST or uncleaved peptide using untrafiltration membrane (Millipore) with a $10 \mathrm{kDa}$ cut-off. The phosphatase activity of $50 \mathrm{nM}-1 \mu \mathrm{M}$ of PP2A core enzyme or holoenzyme containing $\mathrm{B}^{\prime}$ or wild type and mutant PR70 (108-519) was measured using $60 \mu \mathrm{M}$ pCdc6 peptide in a buffer containing 25 $\mathrm{mM}$ Tris $\mathrm{pH} 8.0,150 \mathrm{mM} \mathrm{NaCl}, 3 \mathrm{mM}$ DTT, $50 \mu \mathrm{M} \mathrm{MnCl}_{2}$ and 1 $\mathrm{mM} \mathrm{CaCl} 2$. The reaction was performed in $50 \mu \mathrm{l}$ assay volume at room temperature for $15 \mathrm{~min}$ and stopped by the addition of malachite green $(100 \mu \mathrm{L})$. The absorbance at $620 \mathrm{~nm}$ was measured after $10 \mathrm{~min}$ incubation at room temperature. For steady state kinetics, the assays were performed using $0.16 \mu \mathrm{M}$ of the indicated 
PP2A complexes and titration of pCdc6 peptide (10-380 $\mu \mathrm{M})$. The data were fitted using GraphPad Prism (GraphPad Software Inc.) to calculate $\mathrm{K}_{\mathrm{m}}$ and $\mathrm{K}_{\text {cat }}$.

The phosphatase activity of PP2A samples (0.4-20 nM) toward a universal phosphopeptide substrate (K-R-pT-I-R-R) was measured similarly as previously described [43]. For steady state kinetics, the assays were performed using $0.4 \mathrm{nM}$ of the indicated PP2A complexes and titration of pThr peptide $(75 \mu \mathrm{M}-1.2 \mathrm{mM})$. All experiments were performed in triplicate and repeated three times. Mean \pm SEM were calculated.

\section{Acknowledgments}

We thank Anand Saxena (NSLS) and David Smith (APS LSCAT) for assistance with X-ray diffraction data collection, and Drs Yigong Shi (Tsinghua University) and David Brautigan (University of Virginia) for discussion. This work was supported by the startup fund of the University of Wisconsin-Madison (Y Xing), NIH grants R01 GM096060-01 (Y Xing) and GM86560 (MC Mumby), and T32 CA009135 (N Wlodarchak). The atomic coordinates of the isolated PR72 and the PR70 holoenzyme were deposited in the Protein Data Bank with accession codes 4I5J, 4I5K, 4I5L, 4I5N.

\section{References}

1 Hunter T. Protein kinases and phosphatases: the yin and yang of protein phosphorylation and signaling. Cell 1995; 80:225236.

2 Wurzenberger C, Gerlich DW. Phosphatases: providing safe passage through mitotic exit. Nat Rev Mol Cell Biol 2011; 12:469-482.

3 Janssens V, Goris J. Protein phosphatase 2A: a highly regulated family of serine/threonine phosphatases implicated in cell growth and signalling. Biochem J 2001; 353(Part 3):417-439.

4 Virshup DM. Protein phosphatase 2A: a panoply of enzymes. Curr Opin Cell Biol 2000; 12:180-185.

5 Shi Y. Serine/threonine phosphatases: mechanism through structure. Cell 2009; 139:468-484.

6 Ahn JH, Sung JY, McAvoy T, et al. The B"/PR72 subunit mediates Ca2+-dependent dephosphorylation of DARPP-32 by protein phosphatase 2A. Proc Natl Acad Sci USA 2007; 104:9876-9881.

7 Creyghton MP, Roel G, Eichhorn PJ, et al. PR130 is a modulator of the Wnt-signaling cascade that counters repression of the antagonist Naked cuticle. Proc Natl Acad Sci USA 2006; 103:5397-5402.

8 Creyghton MP, Roel G, Eichhorn PJ, et al. PR72, a novel regulator of Wnt signaling required for Naked cuticle function. Genes Dev 2005; 19:376-386.

9 Hall DD, Feekes JA, Arachchige Don AS, et al. Binding of protein phosphatase 2A to the L-type calcium channel Cav1.2 next to Ser1928, its main PKA site, is critical for Ser1928 dephosphorylation. Biochemistry 2006; 45:3448-3459.

10 Davare MA, Horne MC, Hell JW. Protein phosphatase 2A is associated with class C L-type calcium channels (Cav1.2) and antagonizes channel phosphorylation by cAMP-dependent protein kinase. J Biol Chem 2000; 275:39710-39717.

11 Sablina AA, Hector M, Colpaert N, Hahn WC. Identification of PP2A complexes and pathways involved in cell transforma- tion. Cancer Res 2010; 70:10474-10484.

12 Magenta A, Fasanaro P, Romani S, et al. Protein phosphatase 2A subunit PR70 interacts with $\mathrm{pRb}$ and mediates its dephosphorylation. Mol Cell Biol 2008; 28:873-882.

13 Yan Z, Fedorov SA, Mumby MC, Williams RS. PR48, a novel regulatory subunit of protein phosphatase $2 \mathrm{~A}$, interacts with Cdc6 and modulates DNA replication in human cells. Mol Cell Biol 2000; 20:1021-1029.

14 Davis AJ, Yan Z, Martinez B, Mumby MC. Protein phosphatase $2 \mathrm{~A}$ is targeted to cell division control protein 6 by a calcium-binding regulatory subunit. J Biol Chem 2008; 283:1610416114.

15 Bell SP, Dutta A. DNA replication in eukaryotic cells. Annu Rev Biochem 2002; 71:333-374.

16 Duncker BP, Chesnokov IN, McConkey BJ. The origin recognition complex protein family. Genome Biol 2009; 10:214.

17 Harper JW, Burton JL, Solomon MJ. The anaphase-promoting complex: it's not just for mitosis any more. Genes Dev 2002; 16:2179-2206.

18 Mailand N, Diffley JF. CDKs promote DNA replication origin licensing in human cells by protecting $\mathrm{Cdc} 6$ from $\mathrm{APC} / \mathrm{C}-$ dependent proteolysis. Cell 2005; 122:915-926.

19 Ruediger R, Hentz M, Fait J, Mumby M, Walter G. Molecular model of the A subunit of protein phosphatase 2A: interaction with other subunits and tumor antigens. $J$ Virol 1994; 68:123129.

20 Ruediger R, Roeckel D, Fait J, et al. Identification of binding sites on the regulatory A subunit of protein phosphatase $2 \mathrm{~A}$ for the catalytic $\mathrm{C}$ subunit and for tumor antigens of simian virus 40 and polyomavirus. Mol Cell Biol 1992; 12:4872-4882.

21 Xing Y, Xu Y, Chen Y, et al. Structure of protein phosphatase 2A core enzyme bound to tumor-inducing toxins. Cell 2006; 127:341-353.

$22 \mathrm{Xu} \mathrm{Y,} \mathrm{Chen} \mathrm{Y,} \mathrm{Zhang} \mathrm{P,} \mathrm{Jeffrey} \mathrm{PD,} \mathrm{Shi} \mathrm{Y.} \mathrm{Structure} \mathrm{of} \mathrm{a} \mathrm{pro-}$ tein phosphatase 2A holoenzyme: insights into B55-mediated Tau dephosphorylation. Mol Cell 2008; 31:873-885.

23 Cho US, Xu W. Crystal structure of a protein phosphatase 2A heterotrimeric holoenzyme. Nature 2007; 445:53-57.

$24 \mathrm{Xu} \mathrm{Y,} \mathrm{Xing} \mathrm{Y,} \mathrm{Chen} \mathrm{Y,} \mathrm{et} \mathrm{al.} \mathrm{Structure} \mathrm{of} \mathrm{the} \mathrm{protein} \mathrm{phospha-}$ tase 2A holoenzyme. Cell 2006; 127:1239-1251.

25 Janssens V, Jordens J, Stevens I, et al. Identification and functional analysis of two Ca2+-binding EF-hand motifs in the B"/ PR72 subunit of protein phosphatase 2A. J Biol Chem 2003; 278:10697-10706.

26 Adams SR, Tsien RY. Preparation of the membrane-permeant biarsenicals FlAsH-EDT2 and ReAsH-EDT2 for fluorescent labeling of tetracysteine-tagged proteins. Nat Protoc 2008; 3:1527-1534.

27 Pollok BA, Heim R. Using GFP in FRET-based applications. Trends Cell Biol 1999; 9:57-60.

28 Hoffmann C, Gaietta G, Bunemann M, et al. A FlAsH-based FRET approach to determine $G$ protein-coupled receptor activation in living cells. Nat Methods 2005; 2:171-176.

29 Ragusa MJ, Dancheck B, Critton DA, et al. Spinophilin directs protein phosphatase 1 specificity by blocking substrate binding sites. Nat Struct Mol Biol 2010; 17:459-464.

30 Saraf A, Oberg EA, Strack S. Molecular determinants for PP2A substrate specificity: charged residues mediate dephosphorylation of tyrosine hydroxylase by the $\mathrm{PP} 2 \mathrm{~A} / \mathrm{B}^{\prime}$ regulatory 
subunit. Biochemistry 2010; 49:986-995.

31 Terrak M, Kerff F, Langsetmo K, Tao T, Dominguez R. Structural basis of protein phosphatase 1 regulation. Nature 2004; 429:780-784.

32 O'Connell N, Nichols SR, Heroes E, et al. The molecular basis for substrate specificity of the nuclear NIPP1:PP1 holoenzyme. Structure 2012; 20:1746-1756.

33 Stanevich V, Jiang L, Satyshur KA, et al. The structural basis for tight control of PP2A methylation and function by LCMT1. Mol Cell 2011; 41:331-342.

34 Virshup DM, Shenolikar S. From promiscuity to precision: protein phosphatases get a makeover. Mol Cell 2009; 33:537545.

35 Otwinowski Z, Minor, W. Processing of X-ray diffraction data collected in oscillation mode. Methods Enzymol 1997; 276:307-326.

36 Ness SR, de Graaff RA, Abrahams JP, Pannu NS. CRANK: new methods for automated macromolecular crystal structure solution. Structure 2004; 12:1753-1761.

37 Winn MD, Ballard CC, Cowtan KD, et al. Overview of the CCP4 suite and current developments. Acta Crystallogr D Biol Crystallogr 2011; 67(Part 4):235-242.

38 de Graaff RA, Hilge M, van der Plas JL, Abrahams JP. Matrix methods for solving protein substructures of chlorine and sulfur from anomalous data. Acta Crystallogr D Biol Crystallogr
2001; 57(Part 12):1857-1862.

39 Pannu NS, Read RJ. The application of multivariate statistical techniques improves single-wavelength anomalous diffraction phasing. Acta Crystallogr D Biol Crystallogr 2004; 60(Pt 1):22-27.

40 Cowtan K. The Buccaneer software for automated model building. 1. Tracing protein chains. Acta Crystallogr D Biol Crystallogr 2006; 62(Part 9):1002-1011.

41 Emsley P, Cowtan K. Coot: model-building tools for molecular graphics. Acta Crystallogr D Biol Crystallogr 2004; 60(Part 12 Part 1):2126-2132.

42 Winn MD, Murshudov GN, Papiz MZ. Macromolecular TLS refinement in REFMAC at moderate resolutions. Methods Enzymol 2003; 374:300-321.

43 Chao Y, Xing Y, Chen Y, et al. Structure and mechanism of the phosphotyrosyl phosphatase activator. Mol Cell 2006; 23:535546.

(Supplementary information is linked to the online version of the paper on the Cell Research website.)

(c) (1) (2) This work is licensed under the Creative Commons Attribution-NonCommercial-ShareAlike 3.0 Unported License. To view a copy of this license, visit http://creativecommons.org/licenses/by-nc-sa/3.0 